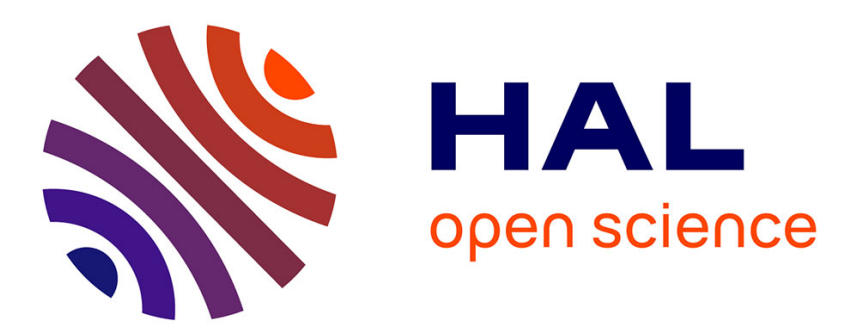

\title{
A density current parameterization coupled with Emanuel's convection scheme. Part I: The models
}

Jean-Yves Grandpeix, Jean-Philippe Lafore

\section{To cite this version:}

Jean-Yves Grandpeix, Jean-Philippe Lafore. A density current parameterization coupled with Emanuel's convection scheme. Part I: The models. Journal of the Atmospheric Sciences, 2010, 67 (4), pp.881-897. 10.1175/2009JAS3044.1 . hal-01135803

\section{HAL Id: hal-01135803 https://hal.science/hal-01135803}

Submitted on 26 Mar 2015

HAL is a multi-disciplinary open access archive for the deposit and dissemination of scientific research documents, whether they are published or not. The documents may come from teaching and research institutions in France or abroad, or from public or private research centers.
L'archive ouverte pluridisciplinaire HAL, est destinée au dépôt et à la diffusion de documents scientifiques de niveau recherche, publiés ou non, émanant des établissements d'enseignement et de recherche français ou étrangers, des laboratoires publics ou privés. 


\title{
A Density Current Parameterization Coupled with Emanuel's Convection Scheme. Part I: The Models
}

\author{
JEAN-YVES GRANDPEIX \\ Laboratoire de Météorologie Dynamique, Paris, France \\ JEAN-PHILIPPE LAFORE \\ CNRM-GAME, Météo-France, and CNRS, Toulouse, France
}

(Manuscript received 22 December 2008, in final form 13 August 2009)

\begin{abstract}
The aim of the present series of papers is to develop a density current parameterization for global circulation models. This first paper is devoted to the presentation of this new wake parameterization coupled with Emanuel's convective scheme. The model represents a population of identical circular cold pools (the wakes) with vertical frontiers. The wakes are cooled by the precipitating downdrafts while the outside area is warmed by the subsidence induced by the saturated drafts. The budget equations for mass, energy, and water yield evolution equations for the prognostic variables (the vertical profiles of the temperature and humidity differences between the wakes and their exterior). They also provide additional terms for the equations of the mean variables. The driving terms of the wake equations are the differential heating and drying due to convective drafts. The action of the convection on the wakes is implemented by splitting the convective tendency and attributing the effect of the precipitating downdrafts to the wake region and the effect of the saturated drafts to their exterior. Conversely, the action of the wakes on convection is implemented by introducing two new variables representing the convergence at the leading edge of the wakes. The available lifting energy (ALE) determines the triggers of deep convection: convection occurs when ALE exceeds the convective inhibition. The available lifting power (ALP) determines the intensity of convection; it is equal to the power input into the system by the collapse of the wakes. The ALE/ALP closure, together with the splitting of the convective heating and drying, implements the full coupling between wake and convection. The coupled wake-convection scheme thus created makes it possible to represent the moist convective processes more realistically, to prepare the coupling of convection with boundary layer and orographic processes, and to consider simulating the propagation of convective systems.
\end{abstract}

\section{Introduction}

During the last three to four decades the understanding of convective processes has improved dramatically. This success is the result of a huge effort by the international atmospheric convection community to patiently combine observation analysis, modeling, and theory. Considerable progress has been made in the description of propagative convection, especially squall lines and, more recently, unorganized convection and the transition from shallow to deep convection. These subjects are akin to some of the challenges met by the

Corresponding author address: J.-Y. Grandpeix, Laboratoire de Météorologie Dynamique, Boite 99, 4, place Jussieu, F-75252, Paris CEDEX 05, France.

E-mail: jyg@Imd.jussieu.fr representation of deep convection in general circulation models (GCMs), namely the representation of convection organization and propagation and of the diurnal cycle of convection over land.

Fast-moving long-lasting squall lines (SLs) provide a convenient archetype of organized mesoscale convective systems (MCSs), facilitating the elaboration of a conceptual model with three components: a convective part, a stratiform part, and a density current (DC). This well-accepted conceptual model is widely described in the literature and confirmed for different tropical and midlatitude regions. The SL appears as a self-maintained system with a loop of interactions: convective cells generate precipitation, which feeds downdrafts by evaporation, which feeds a DC, which spreads at the surface, thus triggering new convective cells. The stratiform part stabilizes the system by favoring mesoscale subsidence 
through evaporation of trailing precipitation under the anvil, reinforcing the DC and a rear-to-front midlevel flow and helping to maintain the "jump updraft" (Moncrieff 1992) in the convective part. From this conceptual model, there are at least three key ingredients to define the degree of organization: the profile of instability measured by the convective available potential energy (CAPE), the low-level shear, and the DC fed by rain evaporation, which is strongly related to the humidity vertical profile whose maximum potential is measured by the DCAPE (downdraft CAPE) concept (see Emanuel 1994). For instance, Global Atmospheric Research Program (GARP) Atlantic Tropical Experiment (GATE) observations revealed a strong link between SL propagation and African easterly jet speed and dryness (Barnes and Sieckman 1984). This link has been confirmed many times from observation and modeling studies and explained by complementary theories. In particular, Rotunno et al. (1988) explain the local matching between the DC propagation and midlevel jet speed in terms of balance between the inflow shear and the vorticity generation by the DC, but other ingredients need be considered, such as SL upstream effect, detrainment from new convective cells when the DC deepens and accelerates, acceleration of the rear inflow (Smull and Houze 1987b; Lafore and Moncrieff 1989), and synoptic-scale effects (Thorpe et al. 1982). A central component of such organized systems is the DC, the properties of which were first studied by Charba (1974) and extensively analyzed by Moncrieff and So (1989).

Other types of MCSs, although less well organized, share the same components and some characteristics of the SL. They all exhibit DCs although their intensity can differ greatly with weaker intensity and depth over oceanic areas, such as those observed during the Tropical Ocean Global Atmosphere Coupled Ocean-Atmosphere Response Experiment (TOGA COARE) (Montmerle et al. 2000), and greater intensity over continents, such as over the Sahel (Redelsperger et al. 2002) or the U.S. Great Plains (Smull and Houze 1987a). Because of their intensity, extension, frequency, and duration (up to 12-36 h) MCSs have a significant large-scale impact (Laing and Fritsch 1997).

Another theory has been developed to explain the organization of isolated convection. Through cloud-resolving model (CRM) simulations of storm development in an idealized environment, Weisman and Klemp (1982) relate the storm type-single cell, multicell, or supercell-to a bulk Richardson number as the ratio between convective instability and the low-level shear. It should be noted that in all these simulations, the surface gust front associated with the DC leading edge is a key feature to explain the storm organization and propagation.
Less organized and shallow convection regimes are much more frequent, so they significantly contribute to heat and moisture diabatic sources. Also, they represent a key stage in the proper simulation of the diurnal cycle of convection and the preparation of the more organized and intense stage of convection. Recent high-resolution simulations of such regimes and of the shallow-to-deep transition highlight the role played by DC in triggering new convective cells and supporting the growth of deep clouds (Tompkins 2001; Khairoutdinov and Randall 2006) through both dynamical and thermodynamic mechanisms depending on the shear occurrence and intensity.

From the above brief overview of our knowledge of convective processes, it appears that DCs play a key role in convection self-maintenance (whether convection is organized or not) and in convection organization and propagation. In spite of this, DCs are rarely represented in current large-scale models: to our knowledge, the pioneering work by Qian et al. (1998, hereafter QYF) has been the only parameterization to represent convective wakes in GCMs. QYF aimed to represent squall lines and their life cycle, but without representing their displacements through grid cell boundaries. They pointed out that failure to represent DCs led to an underestimation of the duration of deep convection episodes and to exceedingly weak surface fluxes. Unfortunately, the test of this parameterization by Rozbicki et al. (1999) in a single-column model (SCM) for 12 squall line cases gave the result that "the modeled wakes were biased too shallow, too warm, and too moist" (p. 1358) and that further development was necessary. Nevertheless, these two papers proved the feasibility and the relevance of wake models. They were the main incentive to develop the model presented in this paper.

The propagation of deep convection from one grid cell to another remains an unresolved question. This has important consequences for large-scale models, especially concerning the simulation of the West African monsoon: it causes simulated deep convection to remain in the trough of African easterly waves contrary to observations, where SLs generally propagate westward faster than the waves (Barnes and Sieckman 1984). Hence, it leads to an erroneous simulation of the interaction between waves and moist convection.

Piriou et al. (2007) emphasized the role of DCs in the diurnal cycle of convection over land: after the initial phase of shallow convection and the transition to deep convection, DCs generated by the evaporation of precipitation grow gradually and, through the uplift of air at the gust fronts, induce a gradual growth of deep convection, leading to a maximum in the afternoon or in the evening. Current large-scale models, in contrast, simulate a maximum of convection close to noon (Yang and 
Slingo 2001). This deficiency appears to be (at least partially) linked to the lack of parameterization of DCs: when the convection scheme depends solely on largescale variables, convection is maximum when the atmospheric instability is maximum (i.e., about noon). It is only thanks to the DCs that convection becomes selfsustaining, which enables it to behave independently of the diurnal heating by the surface. This was well illustrated by Rio et al. (2009), where the simultaneous use of a boundary layer scheme representing shallow convection and of the wake scheme presented in the present paper made it possible to simulate with an SCM a diurnal cycle of moist convection close to the one simulated by CRMs. However, the analysis of the role of DCs in the diurnal cycle of moist convection in GCM simulations remains to be achieved.

The main idea of the present work is that some of the above GCM failures are partly rooted in the fact that the wake subgrid feature and its role are not represented. Thus, this series of papers aims to develop and test a new wake parameterization. The overall structure of QYF's scheme is kept: (i) the domain is split into two regions, the wake and the off-wake regions; (ii) the convective columns develop in the off-wake region, while the precipitating downdrafts are located in the wake region and cool it. However, our new wake parameterization differs from QYF in two respects: (i) whereas QYF considered a single wake inside the grid cell, we consider a population of identical wakes; and (ii) whereas QYF assumed the wake to consist of a mixed layer capped by a sharp inversion, we use a prognostic model of the vertical profiles of the wake temperature and humidity deficits. To express the effect on convection of the lifting at the wake leading edge, much effort has been put into the design of a new convective trigger and closure. We implemented the new trigger and closure in Emanuel's convective scheme (Emanuel 1991, 1993; Hourdin et al. 2006).

The present paper is devoted to the presentation of this new wake parameterization coupled with the Emanuel convective scheme; its evaluation in a single-column framework will be performed in a companion paper (Grandpeix et al. 2010, hereafter Part II) by comparison with well-documented convective case studies exhibiting intense to weak wakes. The formulation of the wake model is presented in section 2; its set of equations for mass, energy, and momentum is derived in section 3 . Section 4 treats the question of the coupling of the wake with the Emanuel convection scheme, which leads us to implement a new trigger function and closure based on the lifting energy and power concepts, respectively. Finally, we summarize and discuss the main characteristics of this new wake scheme and expected progress in section 5 .

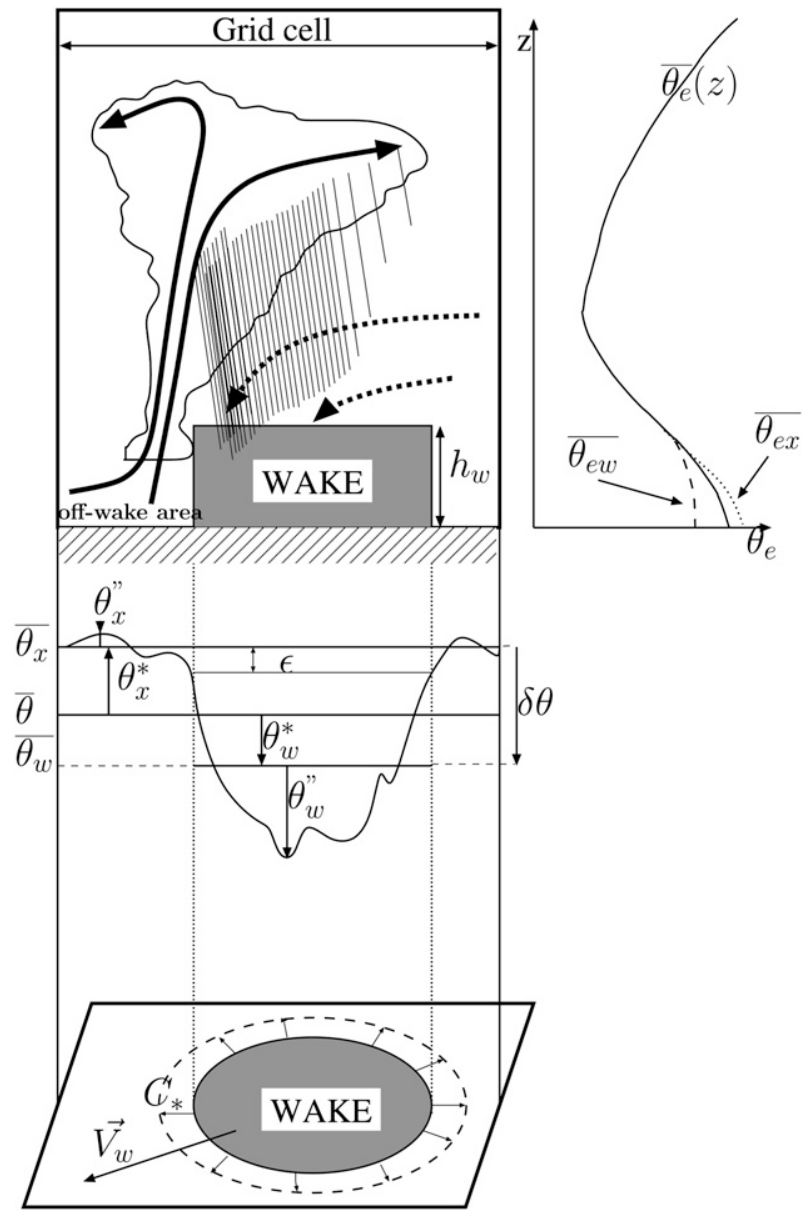

FIG. 1. Sketch of the circular wake model (with one single wake within the grid cell) and of the expected vertical profiles of equivalent potential temperature $\theta_{e}$ averaged over the whole grid cell, the wake region, and the off-wake region.

\section{Wake model}

\section{a. Assumptions and conceptual model}

Figure 1 provides a schematic diagram to help the understanding of our wake model and its physical basis. We consider a population of circular wakes with vertical frontiers over an infinite plane containing the grid cell.

As depicted in Fig. 1, each grid cell is decomposed into two regions: the wake region ( $w$ ) (shaded) and the offwake region (outside the wakes) $(x)$. The wakes are cooled by the convective precipitating downdrafts, while the air outside the wakes feeds the convective saturated drafts. Wake air being denser than off-wake air, wakes spread as density currents. We assume that the wakes have a circular shape, which agrees with observations and CRM simulations at least for the early stage of their development (Fig. 7 of Diongue et al. 2002). The wake shape becomes more ovoid later for mature convective 
systems in a sheared environment. However, we neglect this process, and we assume that the wake geometrical changes with time are only due to their mean movement relative to the ground (velocity $\mathbf{V}_{w}$ ), to their spread (speed $C_{*}$ ), and to their coalescence. All the wakes are assumed identical; that is, they have the same height $\left(h_{w}\right)$, radius $(r)$, and vertical profiles of thermodynamic variables. The wake top corresponds to the level at which wake and off-wake temperatures are equal. Below this level, wakes are cold and subside. The vertical profile of the subsiding motion results from the wake spreading at the surface and from the absence of horizontal entrainment and detrainment below the wake top. The temperature difference between the wake and the off-wake regions is reduced by the mass adjustment by gravity waves (GWs); this damping process is zero at the surface, where gravity waves cannot occur, which permits the existence of wakes. Above the surface, it grows with altitude.

The horizontal detrainment from the wakes is assumed to be zero at all levels, and so is the entrainment below wake top. This can appear as a crude approximation, but at this stage of the wake model development we wanted to stay as simple as possible. However, we shall write general equations including detrainment and entrainment processes, which will make it possible to relax the assumptions.

The spatial distribution of wakes is described statistically by a wake density $D_{\mathrm{wk}}(x, y, t)$ of the wake center positions. Then, for a surface element with sufficiently small area $[\delta x, \delta y]$ around $(x, y)$, the probability that there is a wake center in the surface element is $D_{\mathrm{wk}}(x, y, t) \delta x \delta y$. We shall assume that a uniform density $D_{\mathrm{wk}}(t)$ (i.e., a density $D_{\mathrm{wk}}$ independent of $x$ and $y$, but possibly dependent on the grid cell) is sufficient to represent deep convective processes in large-scale models. Then, the wake density $D_{\mathrm{wk}}$ is associated with an average wake spacing $\approx 1 / \sqrt{D_{\mathrm{wk}}}$ and, assuming that wakes merge as soon as they are in contact, a maximum radius $\approx 1 /\left(2 \sqrt{D_{\mathrm{wk}}}\right)$.

A high density $\left(D_{\mathrm{wk}} \approx\right.$ a few $10^{-10} \mathrm{~m}^{-2}$, wake spacing $<100 \mathrm{~km}$, wake radius $<50 \mathrm{~km}$ ) corresponds to several wakes within a grid cell 100 to $300 \mathrm{~km}$ long. It can describe scattered convection as well as the early stage of organized propagating convection. The case of a low density $\left(D_{\mathrm{wk}} \approx 10^{-12} \mathrm{~m}^{-2}\right.$, wake spacing $\approx 1000 \mathrm{~km}$, wake radius $<500 \mathrm{~km}$ ) corresponds to one wake for tens of grid cells. It can describe organized convection, in particular propagating squall lines. In that case, the wakes can become larger than the grid cells after some time: they will no longer be subgrid-scale objects. The processes occurring at the gust front, however, are always subgrid-scale processes, but they occur in only a small proportion of grid cells.
Whatever the density, when the wake radius approaches the maximum radius, coalescence occurs and the wake density $D_{\mathrm{wk}}$ drops. In the present series of papers, we shall impose, somewhat arbitrarily, the density $D_{\mathrm{wk}}(t)$ and leave for further studies the representation of the organization of moist convection by means of models of the density $D_{\mathrm{wk}}$.

Since wakes are randomly distributed, the number of wakes included in the grid cell or intersecting the contour of the cell will vary depending on the configuration. The purpose of the parameterization is to determine the average effect of these various situations on large-scale thermodynamic variables.

\section{b. Notations and some basic formulas}

- Generally, the overbar denotes the average over a horizontal domain, whether it is the whole grid cell (e.g., the average potential temperature at a given level reads $\bar{\theta}$ ), the off-wake region (e.g., $\overline{\theta_{x}}$ ), or the wakes (e.g., $\overline{\theta_{w}}$ ).

- The wake region is the region where $\theta$ is below $\overline{\theta_{x}}-\epsilon$ (Fig. 1), $\epsilon$ being some minimum temperature deficit (e.g., $\epsilon=0.2 \mathrm{~K}$; but we shall use the limit $\epsilon \rightarrow 0$ ).

- The fractional area of the wakes is given by $\sigma_{w} ; S_{w}, S_{x}$, and $S_{t}$ are the wake, the off-wake region, and the grid cell areas, respectively: $\sigma_{w}=S_{w} / S_{t} ; 1-\sigma_{w}=S_{x} / S_{t}$. Note that $\sigma_{w}$ is related to the wake radius $r$ and to the wake density $D_{\mathrm{wk}}$ by the relation

$$
\sigma_{w}=D_{\mathrm{wk}} \pi r^{2}
$$

- For every field $\alpha$, the restriction to the off-wake region (wake region) is denoted $\alpha_{x}\left(\alpha_{w}\right)$. Each of these fields is decomposed into three terms:

$$
\begin{aligned}
& \alpha_{x}=\bar{\alpha}+\alpha_{x}^{*}+\alpha_{x}^{\prime \prime} \\
& \alpha_{w}=\bar{\alpha}+\alpha_{w}^{*}+\alpha_{w}^{\prime \prime},
\end{aligned}
$$

where $\alpha_{x}^{*}$ is the average over the off-wake region of $\alpha_{x}-\bar{\alpha}, \alpha_{w}^{*}$ is the average over the wake region of $\alpha_{w}-$ $\bar{\alpha}$, and $\alpha_{x}^{\prime \prime}$ and $\alpha_{w}^{\prime \prime}$ are the small-scale fluctuation terms (Fig. 1).

- In addition the difference between the wake and the off-wake region averages is denoted $\delta \alpha$ :

$$
\delta \alpha=\alpha_{w}^{*}-\alpha_{x}^{*}=\overline{\alpha_{w}}-\overline{\alpha_{x}} .
$$

We use Cartesian horizontal $=(x, y)$ coordinates and pressure $=p$ in the vertical direction, so that the velocity horizontal components are $=(u, v)$ and $=\omega$ for the vertical. In addition to the GCM state variables $\bar{\theta}(p)$ (the average potential temperature at level $p$ ) and $\overline{q_{v}}(p)$ 
(the average specific humidity at level $p$ ), the wake scheme introduces the new state variables $\delta \theta(p), \delta q_{v}(p)$, and $\sigma_{w}$.

The above definitions allow the following basic equations to be written:

$$
\begin{aligned}
\alpha_{x}^{*} & =\frac{-\sigma_{w}}{1-\sigma_{w}} \alpha_{w}^{*} \\
\delta \alpha & =\frac{\alpha_{w}^{*}}{1-\sigma_{w}}=\frac{-\alpha_{x}^{*}}{\sigma_{w}} \\
\bar{\alpha} & =\sigma_{w} \overline{\alpha_{w}}+\left(1-\sigma_{w}\right) \overline{\alpha_{x}},
\end{aligned}
$$

from which it is straightforward to derive a first evolution equation at the grid cell scale:

$$
\partial_{t} \bar{\alpha}=\sigma_{w} \partial_{t} \overline{\alpha_{w}}+\left(1-\sigma_{w}\right) \partial_{t} \overline{\alpha_{x}}+\delta \alpha \partial_{t} \sigma_{w} .
$$

\section{c. Wake spreading and propagation}

We make the approximation that the contour of each wake follows the fluid in the lowest part of the planetary boundary layer (PBL). More precisely, we assume that the contour velocity $\mathbf{V}_{\Gamma(M)}$ at point $M$ and the horizontal fluid velocity $\mathbf{V}_{1(M)}$ at $M$, averaged over the lowest layer of the PBL, coincide. Above the low layer, the fluid velocity and the contour velocity no longer coincide.

In the present paper we do not need to treat the wake propagation speed $\mathbf{V}_{w}$ because our aim is wake validation in a single-column model. Nevertheless, this speed will later allow the convection to be moved within each grid cell and translated from one GCM column to an adjacent one when the wake reaches the corresponding grid cell boundary. The formulation of the wake propagation will account for the mean wind and for the lowlevel shear as learned from the convection organization studies summarized in the introduction. Here, the convection scheme is simply gradually switched off in the original grid cell as soon as the wake size reaches a threshold $\left(\sigma_{w}=0.8\right)$.

Given that $C_{*}$ is the mean spreading speed of the wake leading edge (Fig. 1), the wake expansion rate is $\partial_{t} \sigma_{w}=2 \pi r C_{*} D_{\mathrm{wk}}$, or, equivalently (since $\sigma_{w}=D_{\mathrm{wk}} \pi r^{2}$ ),

$$
\partial_{t} \sigma_{w}=2 C_{*} \sqrt{\pi D_{\mathrm{wk}} \sigma_{w}} .
$$

The wake potential energy (WAPE) is defined as the mean potential energy deficit in the wake region:

$$
\mathrm{WAPE}=-g \int_{0}^{h_{w}} \frac{\delta \theta_{v}}{\overline{\theta_{v}}} d z
$$

where $\theta_{v}$ is the virtual potential temperature and $h_{w}$ is the mean wake depth (at which $\delta \theta_{v}$ vanishes). Following
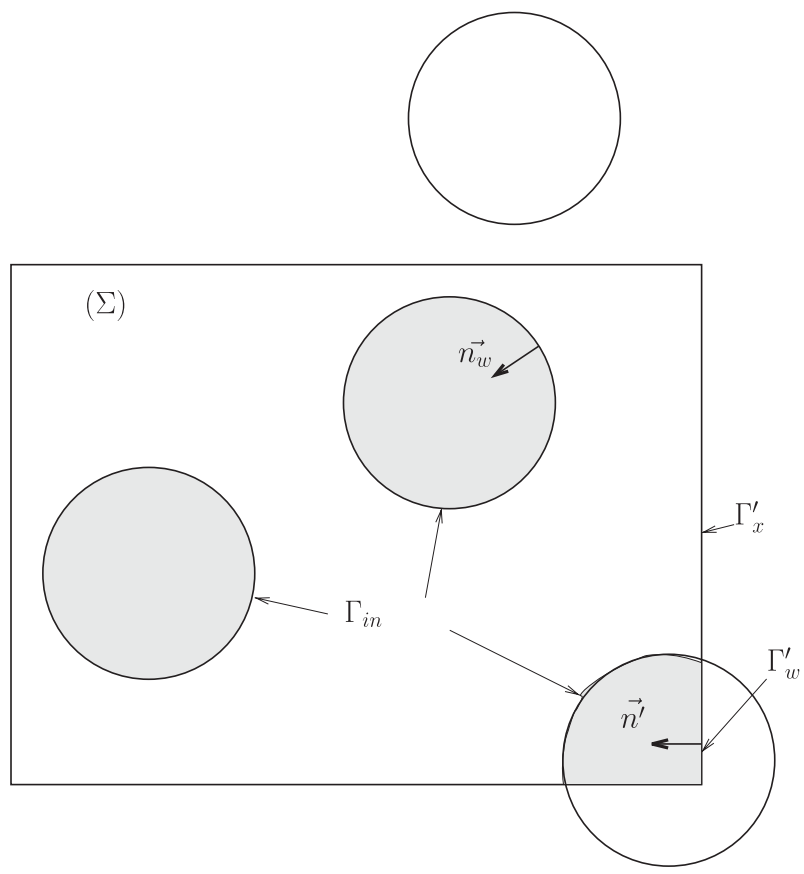

FIG. 2. Notations for a field of wakes extending out of the grid cell $(\Sigma)$ with frontier $\left(\Gamma^{\prime}\right)$. The normals $\mathbf{n}_{w}\left(\mathbf{n}^{\prime}\right)$ to the wake contour $\Gamma_{\text {in }}$ (the grid cell frontier $\Gamma^{\prime}$ ) are oriented inward.

von Kármán (1940), the wake spreading speed is proportional to the square root of this energy:

$$
C_{*}=k_{*} \sqrt{2 \mathrm{WAPE}} .
$$

Here $k_{*}$ is constant and has been estimated at 0.68 (Lafore and Moncrieff 1989) and 0.33 (J. P. Lafore 2000, personal communication) from squall line CRM simulations in $2 \mathrm{D}$ and $3 \mathrm{D}$, respectively. It is consistent with the 0.5 value found by Bryan et al. (2005) from observations of cold pools during the Bow Echo and Mesoscale Convective Vortex Experiment (BAMEX) over the U.S. Great Plains.

\section{Prognostic equation derivations}

We consider the part of a set of wakes that lies inside the grid cell $\Sigma$ as displayed by Fig. 2. The set of all wake contours within $\Sigma$ will be called $\Gamma_{\text {in }}$ and the intersection of the grid cell contour with the wakes $\Gamma_{w}^{\prime}$. Thus, the boundary of the wake region inside the cell is made up of the inner contours $\Gamma_{\text {in }}$ and the outer contours $\Gamma_{w}^{\prime}$.

We shall write budget equations for an atmospheric layer enclosed between the two isobaric surfaces at $p$ and $p-\delta p$. We use the hydrostatic approximation, so the mass per unit area in the layer is assumed to be horizontally uniform. Explicit computations will concern the mass budget equation and the energy budget 
equation for the wake and the off-wake regions. We shall skip the computations relative to water budget equations (since they are similar to the energy ones). This section often uses pairs of equations [one for the $(x)$ and one for the $(w)$ region]. The pairs will be numbered and each equation will be referenced by the pair number and a subscript $w$ or $x$ [e.g., $\left(7_{w}\right)$ points to Eq. (7) relative to the mass budget of the $(w)$ region].

\section{a. Mass budget equations}

Using notations defined in section $2 b$, the mass budget equations for the $(w)$ and $(x)$ regions at a given level $p$ read

$$
\left\{\begin{array}{l}
\partial_{t} S_{w}=-\int_{\Sigma_{w}} \partial_{p}\left(\omega_{w}\right) d \Sigma+\int_{\Gamma_{w, \mathrm{in}}}\left(\mathbf{V}-\mathbf{V}_{\Gamma}\right) \cdot \mathbf{n}_{w} d \Gamma+\int_{\Gamma_{w}^{\prime}} \mathbf{V} \cdot \mathbf{n}^{\prime} d \Gamma \\
\partial_{t} S_{x}=-\int_{\Sigma_{x}} \partial_{p}\left(\omega_{x}\right) d \Sigma-\int_{\Gamma_{w, \mathrm{in}}}\left(\mathbf{V}-\mathbf{V}_{\Gamma}\right) \cdot \mathbf{n}_{w} d \Gamma+\int_{\Gamma_{x}^{\prime}} \mathbf{V} \cdot \mathbf{n}^{\prime} d \Gamma
\end{array} .\right.
$$

Each is the sum of three contributions: the vertical convergence of mass, the mass transport through the wake vertical walls, and the mass transport through the grid cell boundaries into wake (off-wake) regions. The second terms are of opposite sign for the $(w)$ and $(x)$ regions as they correspond to their common frontier $\Gamma_{\text {in }}$. Some assumptions must be made to estimate the last term (i.e., the integral over the grid cell boundary). Two opposite cases are considered here by using a flag $\eta$ :

(i) $\eta=0$ for the confined case, where the wakes do not intersect the grid cell boundary, so that the integral $\int_{\Gamma_{w}^{\prime}} \mathbf{V} \cdot \mathbf{n}^{\prime} d \Gamma$ is obviously zero.

(ii) $\eta \stackrel{w}{=} 1$ for the homogeneous case, where the distribution of wakes extends far outside the grid cell. We assume that wake air and off-wake air enter the grid cell at rates that are proportional to the wake area and the off-wake area respectively. A heuristic probabilistic derivation of this property is given in appendix A. The idea is that if, say, $\sigma_{w}=2 / 3$ and $\sigma_{x}=1 / 3$, then the part $\Gamma_{w}^{\prime}$ of the contour $\Gamma^{\prime}$ will also be twice as long as $\Gamma_{x}^{\prime}$, yielding feeding rates twice as large for the wakes as for the off-wake region. This relation reads

$$
\int_{\Gamma_{w}^{\prime}} \mathbf{V} \cdot \mathbf{n}^{\prime} d \Gamma=S_{w} \partial_{p} \bar{\omega}
$$

Using Eq. (8) and the $\eta$ flag, the mass Eq. $\left(7_{w}\right)$ becomes

$$
\partial_{t} S_{w}=-\int_{\Sigma_{w}} \partial_{p}\left(\omega_{w}-\eta \bar{\omega}\right) d \Sigma+\int_{\Gamma_{w, \text { in }}}\left(\mathbf{V}-\mathbf{V}_{\Gamma}\right) \cdot \mathbf{n}_{w} d \Gamma .
$$

The last integral of this equation represents the horizontal exchange of mass between the regions $(w)$ and $(x)$. It can be split into two parts, corresponding to the entrainment into the wakes and the detrainment from the wakes: let $\Gamma_{\text {in }}^{+}\left(\Gamma_{\text {in }}^{-}\right)$be the part of the contour $\Gamma_{\text {in }}$ where $\left(\mathbf{V}-\mathbf{V}_{\Gamma}\right) \cdot \mathbf{n}_{w}$ is positive (negative). The wake entrainment and detrainment rates $e_{w}$ and $d_{w}$ are then defined by

$$
e_{w}=\frac{1}{S_{t}} \int_{\Gamma_{w, \text { in }}^{+}}\left(\mathbf{V}-\mathbf{V}_{\Gamma}\right) \cdot \mathbf{n}_{w} d \Gamma
$$

and

$$
d_{w}=-\frac{1}{S_{t}} \int_{\Gamma_{w, \mathrm{in}}^{-}}\left(\mathbf{V}-\mathbf{V}_{\Gamma}\right) \cdot \mathbf{n}_{w} d \Gamma
$$

Using the notations of section 2b, Eq. (9) becomes

$$
\frac{\partial_{t} \sigma_{w}}{\sigma_{w}}=-\left(1-\sigma_{w}\right) \partial_{p} \delta \omega-(1-\eta) \partial_{p} \bar{\omega}+\frac{e_{w}-d_{w}}{\sigma_{w}} .
$$

In this equation the effect of large-scale advection is entirely represented by the $\bar{\omega}$ term. This very simple form results from the assumption that the distribution of wakes is either horizontally homogeneous or confined.

Since the large-scale vertical motion $\bar{\omega}$ is known and since the wake spreading rate $\partial_{t} \sigma_{w}$ is given by Eq. (4), Eq. (10) provides a relation between the entrainment/ detrainment rates $e_{w}$ and $d_{w}$ and the vertical velocity difference $\delta \omega$ between the wake and the off-wake regions. This relation will be used in the following section, together with some simple physical hypotheses, to determine $e_{w}, d_{w}$, and $\delta \omega$.

\section{b. Momentum equation: Vertical velocity difference and entrainment}

Equation (10) will be used in two different ways depending on the level considered. Figure 3 illustrates the partition of the troposphere into three layers by two 


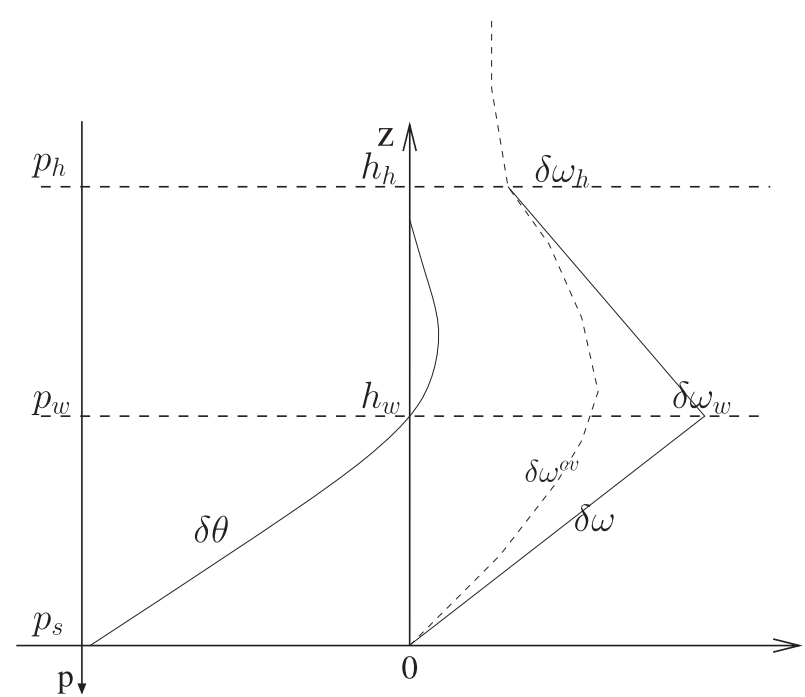

FIG. 3. Expected vertical profiles of $\delta \theta$ and $\delta \omega$ in a case where $e_{w}-d_{w}=0$ below wake top. Both profiles are linear below wake top (pressure $p_{w}$ ); the $\delta \omega^{\mathrm{cv}}$ profile is a sketch of the vertical velocity difference resulting solely from the convective mass fluxes.

horizontal planes: (i) the wake top (altitude $h_{w}$, pressure $\left.p_{w}\right)$, which is the level at which $\delta \theta$ reaches zero, and (ii) the homogeneity level $p_{h}$ (here $0.6 p_{s}$, where $p_{s}$ is the surface pressure), which is the level above which thermodynamic differences between $(w)$ and $(x)$ regions are assumed negligible apart from the convective drafts [saturated drafts in the $(x)$ region, unsaturated downdrafts in the ( $w$ ) region]. The existence of such a level is supported by observations (LeMone and Zipser 1980; Jorgensen and LeMone 1989); it can be explained by the fast mass adjustment by gravity waves in the free atmosphere, especially for the tropics.

Below $h_{w}, \delta \theta$ is negative as we are within the wake layer. Also, the wake boundaries are expected to be nearly impermeable because this ensures the existence of wakes. We thus assume $e_{w}-d_{w}=0$, which is at least true at the surface because of the wake boundary definition.
The vertical integration of Eq. (10) provides the vertical profile of $\delta \omega$ within the wake layer:

$$
\left(1-\sigma_{w}\right) \delta \omega=\left(p_{s}-p\right) \frac{\partial_{t} \sigma_{w}}{\sigma_{w}}-(1-\eta) \bar{\omega},
$$

which increases linearly from $p_{s}$ to $p_{w}$, at least for the homogeneous case $\eta=1$. It corresponds to the mesoscale subsidence (Zipser 1977) associated with the wake region because its spreading rate $\partial_{t} \sigma_{w}$ is positive (Fig. 3).

Above $h_{h}$, the difference $\delta \omega$ between average vertical velocities over $(w)$ and $(x)$ regions is equal to the velocity difference $\delta \omega^{\mathrm{cv}}$ due to the sole convective draft mass fluxes (the precipitating downdraft mass flux $M_{p}$ within the wakes and the net cloud mass flux $M_{c}$ in the off-wake region): $\delta \omega^{\mathrm{cv}}=-g\left\{\left(M_{p} / \sigma_{w}\right)-\left[M_{c} /\left(1-\sigma_{w}\right)\right]\right\}$. Since the two regions are alike, entrainment and detrainment are irrelevant.

Between $h_{w}$ and $h_{h}$, we are still in the mesoscale subsidence fed by evaporation of trailing precipitation represented by the unsaturated downdrafts of the convection scheme. We prescribe the $\delta \omega$ profile as a linear interpolation between $\delta \omega_{h}=\delta \omega^{\mathrm{cv}}\left(p_{h}\right)$ and $\delta \omega_{w}$ as previously diagnosed at $p_{w}$ (Fig. 3). In consequence, Eq. (10) provides the net entrainment rate $e_{w}-d_{w}$, which must be positive in order to feed the expansion of the wakes. Owing to the hypothesis that $d_{w}=0$ everywhere, Eq. (10) yields the following expression for $e_{w}$ above the wake top:

$$
\frac{e_{w}}{\sigma_{w}}=\left(1-\sigma_{w}\right) \partial_{p} \delta \omega+(1-\eta) \partial_{p} \bar{\omega}+\frac{\partial_{t} \sigma_{w}}{\sigma_{w}} .
$$

\section{c. Energy budget equations}

The rate of change of thermal energy of the wake is the sum of the latent heating, the vertical convergence of heat, the heat transport through the wake vertical walls, and the heat transport through the grid cell boundaries into wakes. Similar reasoning applies to the off-wake area. Thus, the energy budget equations read

$$
\left\{\begin{array}{l}
\partial_{t}\left(\int_{\Sigma_{w}} \theta_{w} d \Sigma\right)=\int_{\Sigma_{w}} \frac{\theta_{w}}{T_{w}} \frac{H_{w}}{C_{p}} d \Sigma-\int_{\Sigma_{w}} \partial_{p}\left(\omega_{w} \theta_{w}\right) d \Sigma+\int_{\Gamma_{w, \text { in }}}\left(\mathbf{V}-\mathbf{V}_{\Gamma}\right) \cdot \mathbf{n}_{w} \theta d \Gamma+\int_{\Gamma_{w}^{\prime}} \mathbf{V} \cdot \mathbf{n}^{\prime} \theta d \Gamma \\
\partial_{t}\left(\int_{\Sigma_{x}} \theta_{x} d \Sigma\right)=\int_{\Sigma_{x}} \frac{\theta_{x}}{T_{x}} \frac{H_{x}}{C_{p}} d \Sigma-\int_{\Sigma_{x}} \partial_{p}\left(\omega_{x} \theta_{x}\right) d \Sigma-\int_{\Gamma_{w, \mathrm{in}}}\left(\mathbf{V}-\mathbf{V}_{\Gamma}\right) \cdot \mathbf{n}_{w} \theta d \Gamma+\int_{\Gamma_{x}^{\prime}} \mathbf{V} \cdot \mathbf{n}^{\prime} \theta d \Gamma
\end{array},\right.
$$

where $H_{w}$ and $H_{x}$ represent the heat release (both latent and sensible) in the wake and in the off-wake regions, respectively.
The rest of this section will be devoted to establishing energy equations in an advective form, from which all contour integrals will be removed. 
Subtracting $\overline{\theta_{w}} \times\left(7_{w}\right)$ from Eq. $\left(13_{w}\right)$ and $\overline{\theta_{x}} \times\left(7_{x}\right)$ from Eq. $\left(13_{x}\right)$ yields energy equations in which the contour integrals contain only temperature difference terms:

$$
\left\{\begin{array}{l}
S_{w} \partial_{t} \overline{\theta_{w}}=\int_{\Sigma_{w}} \frac{\theta_{w}}{T_{w}} \frac{H_{w}}{C_{p}} d \Sigma-\int_{\Sigma_{w}}\left[\partial_{p}\left(\omega_{w} \theta_{w}\right)-\overline{\theta_{w}} \partial_{p} \omega_{w}\right] d \Sigma+\int_{\Gamma_{w, \mathrm{in}}}\left(\mathbf{V}-\mathbf{V}_{\Gamma}\right) \cdot \mathbf{n}_{w}\left(\theta-\overline{\theta_{w}}\right) d \Gamma+\int_{\Gamma_{w}^{\prime}} \mathbf{V} \cdot \mathbf{n}^{\prime}\left(\theta-\overline{\theta_{w}}\right) d \Gamma \\
S_{x} \partial_{t} \overline{\theta_{x}}=\int_{\Sigma_{x}} \frac{\theta_{x}}{T_{x}} \frac{H_{x}}{C_{p}} d \Sigma-\int_{\Sigma_{x}}\left[\partial_{p}\left(\omega_{x} \theta_{x}\right)-\overline{\theta_{x}} \partial_{p} \omega_{x}\right] d \Sigma-\int_{\Gamma_{w, \mathrm{in}}}\left(\mathbf{V}-\mathbf{V}_{\Gamma}\right) \cdot \mathbf{n}_{w}\left(\theta-\overline{\theta_{x}}\right) d \Gamma+\int_{\Gamma_{x}^{\prime}} \mathbf{V} \cdot \mathbf{n}^{\prime}\left(\theta-\overline{\theta_{x}}\right) d \Gamma
\end{array} .\right.
$$

To compute the contour integrals, we make the following assumptions: (i) eddy terms are negligible in the contour integrals, so $\theta$ can be replaced by its average value in these integrals; (ii) average values of $\theta$ over $\Gamma_{w}^{\prime}$ and $\Gamma_{x}^{\prime}$ are $\overline{\theta_{w}}$ and $\overline{\theta_{x}}$, respectively (so integrals over the grid cell boundaries are zero); and (iii) the average value of $\theta$ over $\Gamma_{\text {in }}^{+}\left(\Gamma_{\text {in }}^{-}\right)$is $\overline{\theta_{x}}\left(\overline{\theta_{w}}\right)$. This last hypothesis implements a very simple view of the wakes in which eddy effects are neglected and the flow at wake boundaries either does not cross the boundary or is composed of large adiabatic drafts.
With these hypotheses, the integrals over the grid cell contour $\Gamma^{\prime}$ are zero, as are the integrals over $\Gamma_{\text {in }}^{+}$in the $\overline{\theta_{x}}$ equation and the integrals over $\Gamma_{\text {in }}^{-}$in the $\overline{\theta_{w}}$ equation. Using Eq. (10) to express the integral over the contour $\Gamma_{\text {in }}$ and introducing the decompositions

$$
\left\{\begin{array} { l } 
{ \theta _ { w } = \overline { \theta _ { w } } + \theta _ { w } ^ { \prime \prime } } \\
{ \omega _ { w } = \overline { \omega _ { w } } + \omega _ { w } ^ { \prime \prime } }
\end{array} \quad \left\{\begin{array}{l}
\theta_{x}=\overline{\theta_{x}}+\theta_{x}^{\prime \prime} \\
\omega_{x}=\overline{\omega_{x}}+\omega_{x}^{\prime \prime},
\end{array}\right.\right.
$$

we obtain, after some elementary algebra, a first usable form of the energy budget equations for the regions $(x)$ and $(w)$ :

$$
\left\{\begin{array}{l}
\sigma_{w} \partial_{t} \overline{\theta_{w}}=\frac{1}{S_{t}} \int_{\Sigma_{w}}\left[\frac{\theta_{w}}{T_{w}} \frac{H_{w}}{C_{p}}-\partial_{p}\left(\omega_{w}^{\prime \prime} \theta_{w}^{\prime \prime}\right)\right] d \Sigma-\sigma_{w} \overline{\omega_{w}} \partial_{p} \overline{\theta_{w}}-e_{w} \delta \theta \\
\left(1-\sigma_{w}\right) \partial_{t} \overline{\theta_{x}}=\frac{1}{S_{t}} \int_{\Sigma_{x}}\left[\frac{\theta_{x}}{T_{x}} \frac{H_{x}}{C_{p}}-\partial_{p}\left(\omega_{w}^{\prime \prime} \theta_{w}^{\prime \prime}\right)\right] d \Sigma-\left(1-\sigma_{w}\right) \overline{\omega_{x}} \partial_{p} \overline{\theta_{x}}+d_{w} \delta \theta
\end{array} .\right.
$$

The two integrals of the right-hand sides are the diabatic terms; they comprise latent heating, radiation cooling, and eddy transport. We shall identify them with the heat sources $Q_{1, w}^{\prime}$ and $Q_{1, x}^{\prime}$ obtained by decomposing into two parts the apparent heat source $Q_{1}^{\prime}=Q_{1}^{\text {cv }}+$ $Q_{R}+Q_{1}^{\text {bl }}$ due to convection, radiation, boundary layer processes, and surface flux processes (the notation $Q_{1}^{\prime}$ is used here so as to keep the notation $Q_{1}$ for the total apparent heat source, including the source due to wakes):

$$
\begin{aligned}
Q_{1}^{\prime} & =\frac{1}{S_{t}}\left(S_{w} Q_{1, w}^{\prime}+S_{x} Q_{1, x}^{\prime}\right) \\
Q_{1, w}^{\prime} & =\frac{1}{S_{w}} \int_{\Sigma_{w}}\left[\frac{\theta_{w}}{T_{w}} H_{w}-C_{p} \partial_{p}\left(\omega_{w}^{\prime \prime} \theta_{w}^{\prime \prime}\right)\right] d \Sigma \\
Q_{1, x}^{\prime} & =\frac{1}{S_{x}} \int_{\Sigma_{x}}\left[\frac{\theta_{x}}{T_{x}} H_{x}-C_{p} \partial_{p}\left(\omega_{x}^{\prime \prime} \theta_{x}^{\prime \prime}\right)\right] d \Sigma,
\end{aligned}
$$

which yields the equations

$$
\left\{\begin{array}{l}
\partial_{t} \overline{\theta_{w}}=\frac{Q_{1, w}^{\prime}}{C_{p}}-\overline{\omega_{w}} \partial_{p} \overline{\theta_{w}}-\frac{e_{w}}{\sigma_{w}} \delta \theta \\
\partial_{t} \overline{\theta_{x}}=\frac{Q_{1, x}^{\prime}}{C_{p}}-\overline{\omega_{x}} \partial_{p} \overline{\theta_{x}}+\frac{d_{w}}{1-\sigma_{w}} \delta \theta
\end{array} .\right.
$$

These two equations are the final equations giving the evolutions of $\overline{\theta_{w}}$ and $\overline{\theta_{x}}$, provided the apparent heat sources $Q_{1, w}^{\prime}$ and $Q_{1, x}^{\prime}$ and the entrainment/detrainment rates $e_{w}$ and $d_{w}$ are known. Thus, it is sufficient to add equations for $e_{w}$ and $d_{w}$ and the model is complete. However, $\overline{\theta_{w}}$ and $\overline{\theta_{x}}$ cannot be used as state variables, since $\bar{\theta}\left[=\sigma_{w} \overline{\theta_{w}}+\left(1-\sigma_{w}\right) \overline{\theta_{x}}\right]$ is already a state variable of the model. We shall use $\bar{\theta}$ and $\delta \theta$ instead.

Applying Eq. (3) to $\theta$ and substituting the expressions given by Eq. (18) for $\partial_{t} \overline{\theta_{w}}$ and $\partial_{t} \overline{\theta_{x}}$ yields the $\bar{\theta}$ equation, while taking the difference between the two (18) equations yields the $\delta \theta$ equation (in which $\delta Q_{1}^{\prime}=Q_{1, w}^{\prime}-Q_{1, x}^{\prime}$ ):

$$
\left\{\begin{array}{l}
\partial_{t} \bar{\theta}=-\bar{\omega} \partial_{p} \bar{\theta}+\frac{Q_{1}^{\prime}+Q_{1}^{\mathrm{wk}}}{C_{p}} \\
\partial_{t} \delta \theta=-\bar{\omega} \partial_{p} \delta \theta+\frac{\delta Q_{1}^{\prime}+\delta Q_{1}^{\mathrm{wk}}}{C_{p}}-\frac{k_{\mathrm{GW}}}{\tau_{\mathrm{GW}}} \delta \theta
\end{array},\right.
$$

where we have added a supplementary term to account for the mass adjustment by gravity waves $(\mathrm{GW})$ and introduced, besides the heat source $Q_{1}^{\prime}=Q_{R}+Q_{1}^{\text {cv }}+$ $Q_{1}^{\mathrm{bl}}$, the heat source $Q_{1}^{\mathrm{wk}}$ due to the wakes and the corresponding differential source $\delta Q_{1}^{\mathrm{wk}}$ : 


$$
\left\{\begin{array}{l}
\frac{Q_{1}^{\mathrm{wk}}}{C_{p}}=+\left[\partial_{t} \sigma_{w}-\left(e_{w}-d_{w}\right)\right] \delta \theta-\delta \omega \sigma_{w}\left(1-\sigma_{w}\right) \partial_{p} \delta \theta \\
\frac{\delta Q_{1}^{\mathrm{wk}}}{C_{p}}=-\left(\frac{e_{w}}{\sigma_{w}}+\frac{d_{w}}{1-\sigma_{w}}\right) \delta \theta-\delta \omega\left[\partial_{p} \bar{\theta}+\left(1-2 \sigma_{w}\right) \partial_{p} \delta \theta\right]
\end{array} .\right.
$$

\section{d. Discussion of the energy equations}

These equations are independent of the assumptions made for $e_{w}$ and $d_{w}$. Five types of contributions may be identified:

(i) The diabatic sources $Q_{1}^{\prime}$ and $\delta Q_{1}^{\prime}$ provided by the convection, radiation, and boundary layer schemes. The introduction of wakes involved a new diabatic source $\delta Q_{1}^{\prime}$, generating a temperature contrast $\delta \theta$ between the wake and off-wake regions. This source will be detailed in section 4 .

(ii) Large-scale vertical advection that acts both on large-scale temperature and $\delta \theta$.

(iii) The differential vertical advection by $\delta \omega$ (the $\partial_{p} \delta \theta$ and $\partial_{p} \bar{\theta}$ terms) can be explained with the support of the diagram in Fig. 4. It simply means that the vertical velocity difference $\delta \omega$ between the wake $\overline{\omega_{w}}$ and off-wake $\overline{\omega_{x}}$ regions induces a change of both the mean temperature and the wake temperature deficit. Since the wake temperature deficit decreases with altitude (i.e., $\left.\partial_{p} \delta \theta<0\right)$ and $\delta \omega$ is downward (Fig. 3), this term raises the grid cell mean temperature at the rate $\sigma_{w}\left(1-\sigma_{w}\right)$, which is maximum at $\sigma_{w}=1 / 2$ and vanishes at $\sigma_{w}=0$ and 1 . It is more difficult to physically explain its effects on the wake temperature deficit with the Eq. (20) formulation. It is better to go back to its equivalent formulation as the difference of vertical advection between the wake and the off-wake regions: $-\delta \omega\left[\partial_{p} \bar{\theta}+\right.$ $\left.\left(1-2 \sigma_{w}\right) \partial_{p} \delta \theta\right]=\omega_{x}^{*} \partial_{p} \overline{\theta_{x}}-\omega_{w}^{*} \partial_{p} \overline{\theta_{w}}$. From Fig. 4 it is trivial to understand that subsidence in the wake will heat it, whereas the upward motion due to mass conservation will cool the off-wake region slightly, so that the wake collapse will reduce its depth and its temperature deficit, except at the surface where no vertical advection can occur.

(iv) Horizontal wake spread and entrainment/ detrainment at the vertical boundaries of the wake region (the $\delta \theta$ terms). The wake spread acts only on the mean temperature as a cooling source. The entrainment/detrainment also lowers (raises) the mean temperature where entrainment is stronger (weaker) than detrainment. In contrast, entrainment and detrainment act to damp the wake $\delta \theta$, as their sum weighted by the area is always positive. (v) Damping by gravity waves $-\left(k_{\mathrm{GW}} / \tau_{\mathrm{GW}}\right) \delta \theta$, which acts only on $\delta \theta$. The damping time is estimated by $\tau_{\mathrm{GW}}=L_{\mathrm{GW}} / C_{\mathrm{GW}} ; C_{\mathrm{GW}}=4 z N$ is the velocity of gravity waves, $N$ is the Brunt-Väisälä frequency, and $L_{\mathrm{GW}}=\sqrt{\sqrt{\sigma_{w}}\left(1-\sqrt{\sigma_{w}}\right) / \sqrt{D_{\mathrm{wk}}}}$ is an estimate of the distance that gravity waves have to span in order to homogenize temperatures. Note that $\tau_{\mathrm{GW}}$ decreases with altitude because of the GW speed depending on the stability $N$ and the maximum vertical wavelength $(4 z)$, both of which increase in the vertical direction. Here $k_{\mathrm{GW}}$ is a tunable coefficient on the order of 1 .

\section{e. Final set of equations}

The set of equations of the proposed wake parameterization is now complete. In short, it consists of the following:

- four prognostic variables that are functions of the altitude \{potential temperature (specific humidity) average $\bar{\theta}(p)\left[\overline{q_{v}}(p)\right]$ and difference $\left.(w)-(x) \delta \theta(p)\left[\delta q_{v}(p)\right]\right\}$ and corresponding equations [Eq. (19) and the analogous equations for specific humidity];

- a prognostic variable $\sigma_{w}$ [Eq. (4)];

- two diagnostic variables that are functions of the altitude $\delta \omega(p)$ [Eq. (12)] and $e_{w}(p)$ [Eq. (13)], and we assume $d_{w}=0$ at all levels;

- three free parameters: the coefficient $k_{*}$ linking CAPE and $C_{*}$, the density of wakes $D_{\mathrm{wk}}$ and the coefficient $\eta$ switching between the homogeneous case and the confined case; and

- the intermediate variables $h_{w}$ (or $p_{w}$ ), WAPE and $C_{*}$, which are diagnosed from the profiles $\delta \theta(p), \delta q_{v}(p)$ and Eq. (5).

It should be noted that this set of equations is general in the sense that it does not depend on the convective scheme that is used. In contrast, section 4, below, treats the coupling of the present scheme with a specific convective scheme, namely the Emanuel one. Therefore, the proposed solution and corresponding equations for the coupling developed in section 4 cannot be used for other convective schemes without some adaptations specific to each scheme. 


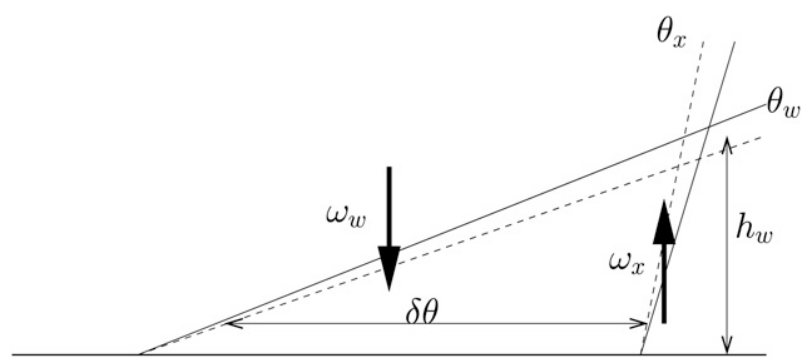

FIG. 4. Diagram to explain the effect of wake collapse.

\section{Coupling of the wake with the convection scheme}

To implement the two-way coupling between wakes and deep convection, we first describe the action of deep convection on wakes, which amounts to expressing the source terms $\delta Q_{1}^{\mathrm{cv}}$ and $\delta Q_{2}^{\mathrm{cv}}$ (section $4 \mathrm{a}$ ). Then we represent the action of wakes on convection. Our fundamental hypothesis is that the dependence of convection on density currents is twofold. First, the presence of density currents creates a dual environment for the convective drafts: the convective saturated drafts lie in the unperturbed region whereas the unsaturated downdrafts lie in the wake regions. Second, the lifting of air at the gust fronts controls the occurrence and the intensity of convection. Taking account of the first item in the convective parameterization is straightforward (section $4 b)$. Accounting for the lifting at the gust front is more involved and requires the design of a new trigger and closure (section 4c).

\section{a. Wake feeding by the convection scheme}

In Emanuel's scheme, $\delta Q_{1}^{\text {cv }}$ is made up of four terms [Eq. (27) of Emanuel 1991]: (i) heating due to the saturated draft compensating subsidence $\left(\mathcal{Q}_{1}^{S}=-g M_{c} \partial_{p} \bar{\theta}\right.$, where $M_{c}$ is the net cloud mass flux); (ii) heating due to detrainment from the cloud $\left(\mathcal{Q}_{1}^{D}\right)$; (iii) heating due to turbulent fluxes associated with unsaturated downdrafts $\left(\mathcal{Q}_{1}^{T}=-g \partial_{p}\left[M_{p}\left(\theta_{p}-\bar{\theta}\right)\right]\right.$, where $M_{p}$ and $\theta_{p}$ are the precipitating downdraft mass flux and potential temperature, respectively); and (iv) latent heating due to precipitation melting and evaporation $\left(\mathcal{Q}_{1}^{E}\right)$. In the present configuration the saturated drafts and their heating effects are located within the $x$ region, while the unsaturated downdrafts and their effects are located within the $w$ region. Thus, $Q_{1}^{\mathrm{cv}}$ and $\delta Q_{1}^{\mathrm{cv}}$ are given by

$$
\begin{aligned}
Q_{1}^{\mathrm{cv}} & =\mathcal{Q}_{1}^{S}+\mathcal{Q}_{1}^{D}+\mathcal{Q}_{1}^{T}+\mathcal{Q}_{1}^{E} \\
\mathcal{Q}_{1}^{S} & =-g M_{c} \partial_{p} \overline{\theta_{x}} \\
\mathcal{Q}_{1}^{T} & =-g \partial_{p}\left[M_{p}\left(\theta_{p}-\overline{\theta_{w}}\right)\right],
\end{aligned}
$$

$$
\begin{aligned}
Q_{1}^{\mathrm{cv}} & =\sigma_{w} Q_{1, w}^{\mathrm{cv}}+\left(1-\sigma_{w}\right) Q_{1, x}^{\mathrm{cv}} \\
\sigma_{w} Q_{1, w}^{\mathrm{cv}} & =\mathcal{Q}_{1}^{T}+\mathcal{Q}_{1}^{E} \\
\left(1-\sigma_{w}\right) Q_{1, x}^{\mathrm{cv}} & =\mathcal{Q}_{1}^{S}+\mathcal{Q}_{1}^{D} \\
\delta Q_{1}^{\mathrm{cv}} & =Q_{1, w}^{\mathrm{cv}}-Q_{1, x}^{\mathrm{cv}} .
\end{aligned}
$$

\section{b. A dual environment for the convection scheme}

The effect of the dual environment is twofold. First, the stability profile seen by saturated drafts and the water vapor they entrain is shielded from the stabilizing effect of the unsaturated downdrafts. Second, the unsaturated downdrafts entrain air from the wake regions, which leads to a subtle interplay between the wakes and the precipitating downdrafts. The wake temperature and humidity result partly from the evaporative cooling and moistening by the downdrafts; conversely, the strength of the downdrafts depends on the relative humidity of the wake regions. This interplay between wakes and downdrafts induces many feedbacks, which greatly influence the time for which convective systems persist.

\section{c. The closure and trigger modifications to account for the wake}

In the standard version of the GCM of the Laboratoire de Météorologie Dynamique, with zoom capability (LMDZ4; Hourdin et al. 2006), as in all GCMs we know of, the convective trigger and closure are functions solely of large-scale variables. Especially in LMDZ4 closure, which is similar to the closure of Emanuel (1993), the cloud-base mass flux $M$ is roughly a function of CAPE and proportional to the square of the buoyancy $B$, at some prescribed altitude above the lifting condensation level (LCL), of adiabatically lifted parcels from the first model level. The trigger consists simply in $B$ being larger than a given threshold.

These formulations of trigger and closure are in accordance with the hypothesis that deep convection is in quasi-equilibrium with the large-scale environment. However, in the presence of density currents and high convective inhibition (CIN), the convection is no longer in quasi-equilibrium with the large scale. This situation translates into the impossibility of expressing convection as a function of the large-scale variables alone. When $|\mathrm{CIN}|=50 \mathrm{~J} \mathrm{~kg}^{-1}$, for instance, convection may occur in the presence of density currents, while it is impossible otherwise; this is a contrasted behavior that cannot be represented in terms of large-scale variables. Thus, some extra variables have to be introduced in order to represent the effect of density currents on deep convection. As emphasized in the introduction and by many authors (e.g., Emanuel 1997), the process to be accounted for by 


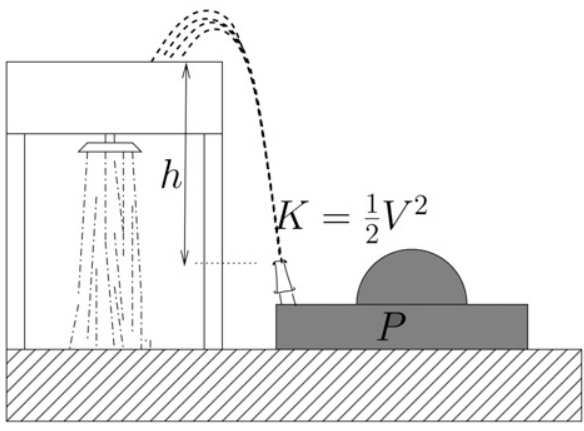

(a)

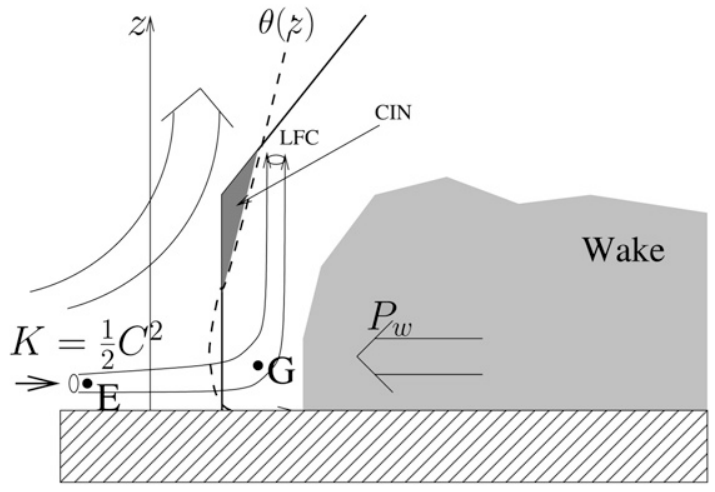

(b)

FIG. 5. Analogy between a pump having to feed a water spout and a density current enabling deep convection. (a) The pump (power $P$ ) yields a mass flow rate $M$ with a kinetic energy $K$ at the outlet; a fraction $k$ (the engine efficiency) of $P$ is converted into the stream power $M K$. The shower is triggered when $K>g h$. The mass flow rate is given by $M=k P / K$. (b) The wakes provide a power $P_{w}$ [the ALP; Eq. (25)] and lift air impinging on the gust front with maximum kinetic energy $K=(1 / 2) C_{\max }^{2}$ (the ALE). Convection is triggered when $K>|\mathrm{CIN}|$. The updraft mass flux is given by Eq. (33), which is similar to the formula giving the mass flow rate for the spout (except for the denominator, which is a bit more complicated).

these supplementary variables is the lifting at the gust front.

We shall introduce two extra variables: (i) the available lifting energy (ALE), which determines the occurrence of convection, and (ii) the available lifting power (ALP), which yields the intensity of convection. The necessity for at least two variables can be understood from the analogy with a pump feeding a water spout that has to reach a given height (Fig. 5). The possibility of reaching the required height is controlled by the velocity of the stream as it leaves the nozzle, while the mass flow rate is a function of the power of the pump. Similarly, we shall argue that the possibility that some updrafts may overcome the convective inhibition is controlled by the kinetic energy of the air impinging on the gust front (the ALE), while the cloud-base mass flux of deep convective updrafts is proportional to the power input into the system by the wakes (the ALP).

\section{1) LIFTING ENERGY AND TRIGGER}

To express the lifting energy ALE provided by a density current spreading at the surface, we only use the quite realistic steadiness assumption of the lifting process in the reference frame moving at the speed $C$ of the density current ( $C$ is a random variable whose mean value is $C_{*}$; its maximum value is estimated by $\left.C_{\max }=\sqrt{2 \mathrm{WAPE}}\right)$. As shown by Haman (1976) or Moncrieff (1981), the Bernoulli equation expressing the conservation of energy allows a conservative quantity to be introduced along each streamline within an organized steady convection

$$
\frac{1}{2} v^{2}+\frac{p^{\prime}}{\rho}-\int_{z_{0}}^{z} B d z
$$

as the sum of the kinetic energy (in the relative frame), the fluctuation of pressure $p^{\prime}$ from a hydrostatic reference state ( $\rho$ is the air density), and the vertical integral (along the streamline) of the buoyancy $B$ from a reference level $z_{0}$. We apply this conservative property to a streamline of the environmental air close to the surface entering the convective system at the relative speed $C$ (Fig. 5b). At its entry point $\mathrm{E}$, as the environment is hydrostatic and there is already no buoyancy, the conservative variable is reduced to the kinetic energy $1 / 2 C^{2}$. When this streamline reaches the density current leading edge or gust point $\mathrm{G}$, all the above kinetic energy is converted into pressure fluctuation $p^{\prime}$. This pressure jump deflects the streamline and accelerates it upward to the level of free convection (LFC) against the CIN barrier. At this level, we consider that the pressure is equal to that of the environment, so that the energy conservation property written at these three points $\mathrm{E}, \mathrm{G}$, and $\mathrm{LFC}$ reads

$$
\frac{1}{2} C^{2}=p^{\prime}=K_{\mathrm{LFC}}+|\mathrm{CIN}|
$$

Since $K_{\mathrm{LFC}}$ is positive, stream tubes reaching LFC are possible if and only if $1 / 2 C^{2}>|\mathrm{CIN}|$. Now, considering the whole range of $C$ values, the condition for some lifted drafts to reach $\mathrm{LFC}$ reads $1 / 2 C_{\max }^{2}>|\mathrm{CIN}|$. 
Hence, choosing $\mathrm{ALE}_{\mathrm{wk}}=$ WAPE yields the expected form for the trigger condition, namely $\mathrm{ALE}_{\mathrm{wk}}>$ $|\mathrm{CIN}|$.

\section{2) LIFTING POWER AND ClOSURE}

First we consider the average lifting power input into their environment by the wakes; then we turn to the power input by the wakes within the grid cell.

\section{(i) Power input by the population of wakes}

Each wake provides energy to the wake environment through the relative movement of the wake leading edge with respect to the wake environment. The rate of the energy supply $U_{\mathrm{in}}^{\mathrm{wk}}$ by a single wake is equal to the kinetic energy flux of air impinging on the wake contour of length $L_{1}=2 \pi r$ moving at velocity $C_{*}$ :

$$
U_{\text {in }}^{\mathrm{wk}}=\frac{1}{2} \rho C_{*}^{3} L_{1} h_{w} .
$$

Thus, the average power per unit area input into the environment by the population of wakes with density $D_{\mathrm{wk}}$ reads

$$
P_{\mathrm{in}}^{\mathrm{wk}}=U_{\mathrm{in}}^{\mathrm{wk}} D_{\mathrm{wk}}=\rho C_{*}^{3} h_{w} \sqrt{\pi \sigma_{w} D_{\mathrm{wk}}} .
$$

The power available for lifting $\left(P_{\mathrm{lift}}^{\mathrm{wk}}\right)$ is a fraction $\mathcal{E}_{\text {lift }}^{\mathrm{wk}}$ (the wake lifting efficiency) of $P_{\mathrm{in}}^{\mathrm{wk}}$ :

$$
P_{\mathrm{lift}}^{\mathrm{wk}}=\mathcal{E}_{\mathrm{lift}}^{\mathrm{wk}} \rho C_{*}^{3} h_{w} \sqrt{\pi \sigma_{w} D_{\mathrm{wk}}} .
$$

The lifting efficiency $\mathcal{E}_{\text {lift }}^{\mathrm{wk}}$ represents several mechanisms preventing the full conversion of the power provided by the wakes into lifting power used by convection: (i) only a part of the wake contours generates sufficient convergence to feed the arc of convective cells; (ii) the wake constitutes a nonrigid obstacle; and (iii) a part of the power input by the wakes is lost in dissipation and inhibition. The first two processes depend strongly on the low-level shear and on the stability of the environment. From these three mechanisms (especially from the first one), one expects the efficiency $\mathcal{E}_{\text {lift }}^{\mathrm{wk}}$ to be on the order of $1 / 4$ or $1 / 3$ when the shear is moderate to strong, whereas the lifting efficiency decreases for weak or null shear regimes. At this stage of the development of the wake scheme (basic formulation and validation tests in 2D for Parts I and II of this paper), and to stay as simple as possible, we keep constant this efficiency. Later when testing moving wakes in 3D, we will introduce a dependency on the shear.

\section{(ii) Power input by the wakes into the grid cell}

If the statistics of the wakes is sufficient (i.e., if $D_{\mathrm{wk}} S_{t} \gg 1$ ), it can be assumed that the lifting power available for deep convection within the grid cell is close to the ALP provided by the whole population of wakes, namely $P_{\text {lift }}^{\mathrm{wk}}$. When $D_{\mathrm{wk}} S_{t}<1$, a situation that occurs when a single squall line is crossing the grid cell, $P_{\text {lift }}^{\mathrm{wk}}$ is no longer relevant. In that case we shall use expectation values conditioned on the presence of a gust front within the grid cell.

The power per unit area provided by the wakes within the grid cell along the length $\widehat{L_{\Gamma}}$ of the contour $\Gamma_{\text {in }}$ (Fig. 2) reads

$$
\widehat{P_{\mathrm{in}}^{\mathrm{wk}}}=\frac{1}{S_{t}} \frac{1}{2} \rho C_{*}^{3} \widehat{L_{\Gamma}} h_{w} .
$$

The unconditional expectation value of $\widehat{L_{\Gamma}}$ is $L_{\Gamma}=$ $L_{1} D_{\mathrm{wk}} S_{t}$, so taking the expectation value of Eq. (26) yields back Eq. (24). Simple probabilistic computations (details in appendix B) yield the following expression for the expectation value of $\widehat{L_{\Gamma}}$ conditioned on the presence of a gust front within the grid cell:

$$
\widetilde{L_{\Gamma}}=\frac{L_{\Gamma}}{1-\exp \left[-D_{\mathrm{wk}} S_{A}(r)\right]},
$$

where $S_{A}(r)$ is the area of the domain $\mathcal{A}$ composed of the points that are at a distance $r$ from at least one grid cell point; $S_{A}(r)$ is a complicated function of $r$ that depends on the grid cell shape. We shall not attempt to write down the full expression for $S_{A}(r)$ but give two simple asymptotic limits:

- When $\pi r^{2} \ll S_{t}$, then $\mathcal{A}$ coincides with the grid cell and $S_{A}(r) \approx S_{t}$. Then $\widetilde{L_{\Gamma}} \approx L_{\Gamma}$ if $D_{\mathrm{wk}} S_{t} \gg 1$ and $\widetilde{L_{\Gamma}} \approx 2 \pi r$ when $D_{\mathrm{wk}} S_{t} \ll 1$.

- When $\pi r^{2} \gg S_{t}$, then $\mathcal{A}$ is a ring of radius $r$ and of width $\sqrt{S_{t}}$; thus, $S_{A}(r) \approx 2 \pi r \sqrt{S_{t}}$. Then necessarily $D_{\mathrm{wk}} S_{t} \ll 1$ and $\widetilde{L_{\Gamma}} \approx \sqrt{S_{t}}$.

Then, from $\widetilde{L_{\Gamma}}$, we obtain the expectation value of $\widehat{P_{\text {lift }}^{\mathrm{wk}}}$ conditioned on the presence of a gust front within the grid cell:

$$
\widetilde{P_{\text {lift }}^{\mathrm{wk}}}=\mathcal{E}_{\text {lift }}^{\mathrm{wk}} \frac{1}{2} \rho C_{*}^{3} h_{w} \widetilde{L_{\Gamma}}
$$

Hereafter $\widetilde{P_{\text {lift }}^{\mathrm{wk}}}$ will be used as the $\mathrm{ALP}_{\mathrm{wk}}$.

(iii) Power used by the convective updraft

Following observations by airplanes flying through cumulus clouds (Cruette et al. 2000), we assume that the updrafts at the base of cumulonimbus clouds have a homogeneous liquid water potential temperature $\theta_{l}$ and total water content $q_{t}$, while their vertical velocity $w$ shows large spatial variability (which ensures the required strong mixing within the updraft) (Fig. 6). The equations will be 


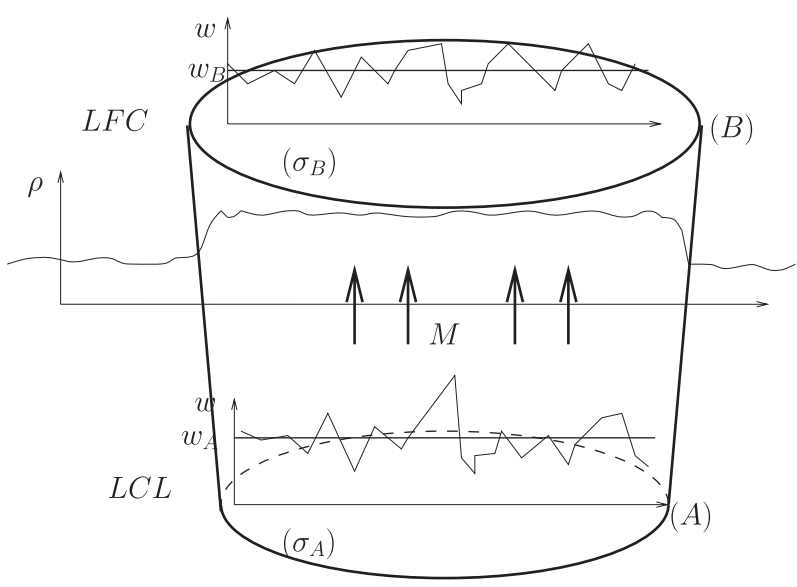

FIG. 6. Convective updraft between cloud base (level A) and level of free convection (level B).

written at two levels. The first level is the condensation level, here called level A; the second level is a level close to the level of free convection but above it, here called level B. The updraft will be considered free of entrainment and shedding between levels A and B. Neglecting entrainment is a fundamental hypothesis of Emanuel's scheme. It may be justified by saying that for the convective column size (a few tens of kilometers) and height (1 or $2 \mathrm{~km}$ ) considered here, entrainment may concern only a thin outer layer of the updraft. Neglecting shedding means that mixed drafts generated close to LCL do not participate in the deep convection process: the model is concerned only with those drafts that actually reach LFC adiabatically. With these hypotheses, updraft mass flux is constant between levels A and B; its value will be called $M$. Then, the fractional area of the updraft varies with the altitude $z$; it will be called $\sigma(z)$, and $\sigma_{A}$ $\left(\sigma_{B}\right)$ is its value at level A (B).

The vertical velocity within the updraft can be decomposed, at each level, into a column average component $\langle w\rangle$ and a fluctuation $w^{\prime \prime \prime}$ :

$$
w(x, y, z)=\langle w\rangle_{z}+w^{\prime \prime \prime}(x, y, z)
$$

(generally, $\langle\cdot\rangle_{z}$ stands for the average over a horizontal section of the updraft at level $z$; in particular, $\langle w\rangle_{B}$ is the average vertical velocity at level B).

The power carried by the updraft at level A is $P_{\text {lift }}^{\mathrm{cv}}$ and the power carried at level $\mathrm{B}$ is $1 / 2 \rho_{B} \sigma_{B}\left\langle w^{3}\right\rangle_{B}$. Assuming steadiness and neglecting dissipation, the difference between these two powers is the (negative) power $P_{\text {buoy }}$ provided by buoyancy forces between levels $\mathrm{A}$ and $\mathrm{B}$ :

$$
P_{\text {buoy }}=\int_{z_{A}}^{z_{B}}\left\langle\rho w g \frac{\theta_{v u}-\theta_{v x}}{\theta_{v x}}\right\rangle_{z} \sigma(z) d z,
$$

where $\theta_{v u}\left(\theta_{v x}\right)$ is the virtual potential temperature of the updraft (the off-wake region). Using the top hat approximation for $\theta$ and $q_{v}$ the power $P_{\text {buoy }}$ reads [since $\langle\rho w\rangle_{z} \sigma(z)=M$ independent of $\left.z\right]$ :

$$
\begin{aligned}
P_{\text {buoy }} & =\int_{z_{A}}^{z_{B}}\langle\rho w\rangle_{z} g \frac{\theta_{v u}-\theta_{v x}}{\theta_{v x}} \sigma(z) d z \\
& =M W_{\text {buoy }},
\end{aligned}
$$

where $W_{\text {buoy }}$ is the work per unit mass of buoyancy forces between levels A and B:

$$
W_{\text {buoy }}=\int_{z_{A}}^{z_{B}} g \frac{\theta_{v u}-\theta_{v x}}{\theta_{v x}} d z .
$$

Then, the energy budget of the updraft between A and $\mathrm{B}$ is

$$
\frac{1}{2} \rho_{B} \sigma_{B}\left\langle w^{3}\right\rangle_{B}=P_{\text {lift }}^{\mathrm{cv}}+W_{\text {buoy }} M .
$$

Assuming isotropic turbulence within the updraft, we can write

$$
\left\langle w^{3}\right\rangle_{B}=\langle w\rangle_{B}^{3}+3\langle w\rangle_{B}\left\langle w^{\prime \prime \prime 2}\right\rangle_{B} .
$$

Hence,

$$
\frac{1}{2} M\langle w\rangle_{B}^{2}+\frac{3}{2} M\left\langle w^{\prime \prime \prime 2}\right\rangle_{B}=P_{\text {lift }}^{\mathrm{cv}}+W_{\text {buoy }} M,
$$

which yields a formula relating the convective mass flux $M$ and the power $P_{\text {lift }}^{\mathrm{cv}}$ carried by the convective updraft at cloud base:

$$
M=\frac{P_{\text {lift }}^{\mathrm{cv}}}{\frac{1}{2}\left(\langle w\rangle_{B}^{2}+3\left\langle w^{\prime \prime \prime 2}\right\rangle_{B}\right)-W_{\text {buoy }}} .
$$

This formula shows that for a given power at cloud base, the convective mass flux is controlled by three quantities: (i) the average velocity of the convective updraft; (ii) the turbulent kinetic energy within the convective updraft; and (iii) the convective inhibition.

In agreement with wind retrieval of Doppler radar observations, 3D CRM simulations of squall lines reveal that $\left\langle w^{\prime \prime \prime 2}\right\rangle$ is on the order of $\langle w\rangle_{B}^{2}$ (see Fig. 5 of Lafore et al. 1988), stressing the highly turbulent character of convective flows. The intensity increases up to midlevels but typical values of $\langle w\rangle_{B}$ at the LFC are in the range of 1 to $2 \mathrm{~m} \mathrm{~s}^{-1}$. Hence, we shall write the first term of the denominator in Eq. (32):

$$
\frac{1}{2}\left(\langle w\rangle_{B}^{2}+3\left\langle w^{\prime \prime \prime 2}\right\rangle\right) \approx 2 w_{B}^{2},
$$

where $w_{B}$ is a tunable parameter. 
(iv) Matching the convective updraft with the wake lifting power

Then, equating the power $P_{\text {lift }}^{\mathrm{cv}}$ used by convective updrafts to cross inhibition with the available lifting power $\widehat{P_{\text {lift }}^{\mathrm{wk}}}$ [given by Eqs. (28) and (27)] yields the ALP closure:

$$
M=\frac{\widetilde{P_{\text {lift }}^{\mathrm{wk}}}}{2 w_{B}^{2}-W_{\text {buoy }}} .
$$

The two free parameters of the closure are as follows:

- the fraction $\mathcal{E}_{\text {lift }}^{\mathrm{wk}}$ of the available lifting power actually used in the updrafts $\left(\mathcal{E}_{\text {lift }}^{\mathrm{wk}}\right.$ should be in the range of 0.1 to 0.5 ), and

- the updraft vertical velocity $w_{B}$ at the free convection level; typically, $w_{B}$ should be in the range of 1 to $2 \mathrm{~m} \mathrm{~s}^{-1}$.

\section{3) INTERFACING WITH EMANUEL'S SCHEME CLOSURE}

Now that an ALP closure has been designed, it is necessary to define a way to interface it with the CAPE closure used in the version of Emanuel's scheme implemented in LMDZ4 GCM.

Consistently with the wake model, the convective model will be considered as representing a population of identical elementary convective systems (ECSs). Then, of course, all tendencies [such as $\left(\partial_{t} T\right)_{\mathrm{cv}}(z)$ ] and other extensive variables (such as the fractional area $\sigma_{d}$ of precipitating downdrafts) will be proportional to the density $D_{c}$ of ECS. In particular, for two situations with same ECS but different densities $D_{c}$ and $D_{c}^{\prime}$, the cloudbase mass fluxes are such that $M / M^{\prime}=D_{c} / D_{c}^{\prime}$.

The standard Emanuel closure of LMDZ4 provides an instance of a population of ECSs evolving in the considered environment with a given fractional area of precipitating downdrafts: $\sigma_{d, 0}=0.01$. The corresponding cloud-base mass flux and tendencies will be denoted $M_{0},\left(\partial_{t} T\right)_{\mathrm{cv}, 0}(z),\left(\partial_{t} q_{v}\right)_{\mathrm{cv}, 0}(z) \ldots$ Then, assuming that the ECS are functions only of the vertical profiles of $T$ and $q_{v}$ and of ALE, the actual tendencies will simply be obtained by scaling

$$
\left(\partial_{t} T\right)_{\mathrm{cv}}(z)=\frac{M}{M_{0}}\left(\partial_{t} T\right)_{\mathrm{cv}, 0}(z) \cdots \sigma_{d}=\frac{M}{M_{0}} \sigma_{d, 0} .
$$

\section{Discussion and conclusions}

The aim of the present series of papers is the development of a wake parameterization for GCMs. This subgrid feature plays a major role in organizing the convection; thus, when coupled with a convection scheme, it is expected to improve it, in particular concerning the diurnal cycle, propagation, and duration. This first paper has been devoted to the presentation of this new wake model coupled with Emanuel's convective scheme. We consider here a population of circular wakes with the same characteristics (radius and height). Their density $D_{\mathrm{wk}}$ can be homogeneous, and extending to infinity, or confined to the grid cell; it is prescribed in the present stage. A final set of wake equations has been derived from the mass, water, and energy budget equations and from simple assumptions for the vertical velocities. It results in two supplementary prognostic variables at each level $p$ for the difference in temperature $\delta \theta(p)$ and specific humidity $\delta q_{v}(p)$ between the wake and the offwake regions. The contributing terms are the diabatic term provided by the convective scheme, the large-scale vertical advection, the subgrid vertical advection due to the wake subsidence, the effect of the wake spreading horizontally, the entrainment/detrainment at the wake frontiers, and the damping effect of gravity waves on $\delta \theta$. Including wakes adds two new terms into the prognostic equations of the mean variables due to the collapse and spread of the wakes. The wakes are characterized by their fractional area $\sigma_{w}$ whose evolution is driven by a prognostic equation. Mass conservation allows the entrainment $e_{w}$ and the difference of vertical velocity $\delta \omega$ between the wake and off-wake regions to be diagnosed. Intermediate variables such as the wake height $h_{w}$ and their deficit in potential energy WAPE are diagnosed from the $\delta \theta(p)$ and $\delta q_{v}(p)$ profiles.

This wake model has been coupled with Emanuel's convection scheme. There is no general rule for coupling this wake model with a convective scheme. Nevertheless, it is possible only if the convective scheme considered represents the unsaturated downdrafts that feed the wake through precipitation evaporation. In that case, the diabatic convective sources are partitioned into two parts: the ones associated with convective drafts fed by the off-wake region and the others associated with the unsaturated downdrafts feeding the wake region.

The wake model interacts strongly with the convection scheme. As seen previously, it first adds diabatic terms. But its key role is in triggering and feeding new convective cells through the convergence generated at the wake leading edge. To account for these processes, we have introduced the new concepts of available lifting energy (ALE) and available lifting power (ALP) for the trigger and closure, respectively. The ALE is proportional to the wake deficit of potential energy (WAPE). Triggering of new convective cells is possible if ALE is stronger than the convective inhibition (CIN) and 
overcomes it. The available lifting power corresponds to the work accomplished by the wake spread. It allows us to derive a formulation of the vertical mass flux at the level of free convection. Keeping the shape of the vertical profile of vertical mass flux provided by the original Emanuel convective scheme, it is possible to close the system.

Emanuel's convective scheme plays a central role in this coupled model. However, the features that are important for the wake-convection coupling are not specific to Emanuel's scheme. Any convection scheme in which a representation of precipitating downdrafts is present can drive the wake scheme. It should be possible, also, to implement the ALP closure in convective schemes using a CAPE closure by using the procedure described at the end of section 4c. However, for schemes that do not use a CAPE closure, such as Emanuel and Zivkovic-Rothman (1999), further work is necessary. Implementing the ALE trigger in other convective schemes might also be more involved; it has to be studied specifically for each convective scheme. Nevertheless, we expect the wake scheme to be coupled with various convective schemes in the coming years.

In Part II, this wake model coupled with Emanuel's convective scheme is widely tested in a single-column framework for two opposite case studies, over the Sahel in West Africa and over the West Pacific oceanic region of TOGA COARE, that exhibit intense and weak wakes, respectively. The sensitivity to the basic parameters of the parameterization is widely explored. Another paper (R. Roehrig and J.-Y. Grandpeix 2009, unpublished manuscript) will describe the implementation of the wake scheme within the LMDZ4 GCM and its testing. The major issues addressed by the related papers are the demonstration of the robustness of the wake model coupled with Emanuel's convective scheme and a verification of its potential to improve the diurnal cycle of convection, its duration, and its maintenance during the night.

This effort to improve the convection parameterization is a long-term task. Different questions need to be addressed in the current and further studies. Concerning the implementation of this scheme in a GCM, the propagation issue must be treated. After diagnosing the convection propagation speed from wake intensity, shear, and mean wind information, we need to find how to move the wake and the convection from one grid cell to the next one. Also, a formulation of the wake lifting efficiency $\mathcal{E}_{\text {lift }}^{\mathrm{wk}}$ as a function of the low-level shear will be introduced and tested to account for the key role played by the shear to organize the convection. Because of wind and thermodynamics differences between the wake and off-wake regions, we also need to treat surface fluxes and PBL in the two regions differently. The resulting enhancement of fluxes by the convective wakes has been studied by Jabouille et al. (1996) and parameterized (Redelsperger et al. 2000). The wake model provides a new way to account for these processes and also allows the wake intensity to be decreased at the surface. Up to now, momentum transport by convection has not been dealt with most of the time. The wake model, allowing the degree of convection organization to be identified, can be used in the future to treat momentum transport. Also, to better feed the wake, it may be important to account for the stratiform part.

The introduction of the ALE and ALP concepts could be a major step toward better treatment of the triggering and closure of convection schemes. Nevertheless, the wake contribution to ALE and ALP is only one contribution among many others. So we need to introduce other processes that contribute to ALE and ALP fields such as PBL, surface heterogeneity, and thermodynamic and dynamical effects of orography and gravity waves. Current and complementary research is underway to further describe ALE and ALP generated by the PBL and the orography. Recently, Rio et al. (2009) coupled this wake model with a PBL scheme and succeeded in simulating the diurnal cycle of convection for an Atmospheric Radiation Measurement (ARM) program case. It will be a decisive contribution allowing us to determine the ALE and ALP fields necessary to represent the convection during its onset, before the wakes take the lead.

Acknowledgments. The European West African Monsoon Project (WAMP) headed by Chris Thorncroft provided the opportunity to meet two different communities working on processes and on GCMs. The initial idea of developing a wake parameterization emerged from this meeting. We thank many colleagues at LMD and CNRM-GAME including Sandrine Bony, Benjamin Diviné, Frederic Hourdin, Alain Lahellec, Catherine Rio, Romain Roehrig, and others for numerous discussions and their comments throughout the development and the implementation of this new scheme. Susan Becker deserves many thanks for the English editing of the text; if there are still some errors, they were added afterward by us. Thanks also to the anonymous reviewers for their useful and pertinent comments that helped to improve the presentation of this series of papers.

\section{APPENDIX A}

\section{Estimation of the Large-Scale Terms in the Mass Budget Equations}

We want to compute the expectation value of $\int_{\Gamma_{w}^{\prime}} \mathbf{V} \cdot \mathbf{n}^{\prime} d \Gamma$. We consider circular wakes with radius $r$ 
and we make the very crude assumption that the velocity field $\mathbf{V}$ is independent of the wake positions. Let $\mathbf{C}_{i}$ represent the centers of the wakes. The integral reads

$$
\int_{\Gamma_{w}^{\prime}} \mathbf{V} \cdot \mathbf{n}^{\prime} d \Gamma=\int_{\Gamma^{\prime}} d \Gamma_{(\mathbf{M})}\left(\mathbf{V} \cdot \mathbf{n}^{\prime}\right)_{(\mathbf{M})} \sum_{i} I\left(\frac{\left\|\mathbf{M}-\mathbf{C}_{i}\right\|}{r}\right),
$$

where the function $I$ is defined by $I(x)=1$ if $0<x<1$ and $I(x)=0$ otherwise.

The expectation value of the integral is

$$
\begin{aligned}
& \left\langle\int_{\Gamma_{w}^{\prime}} \mathbf{V} \cdot \mathbf{n}^{\prime} d \Gamma\right\rangle \\
& \quad=\int_{\Sigma^{\prime}} d^{2} C D_{w(\mathbf{C})} \int_{\Gamma^{\prime}} d \Gamma_{(\mathbf{M})}\left(\mathbf{V} \cdot \mathbf{n}^{\prime}\right)_{(\mathbf{M})} I\left(\frac{\|\mathbf{M}-\mathbf{C}\|}{r}\right),
\end{aligned}
$$

where $\left(\Sigma^{\prime}\right)$ is a domain much larger than $(\Sigma)$.

In the particular case where $D_{w(\vec{C})}$ is uniform, the $C$ integration is trivial. One gets

$$
\begin{aligned}
\left\langle\int_{\Gamma_{w}^{\prime}} \mathbf{V} \cdot \mathbf{n}^{\prime} d \Gamma\right\rangle & =\pi r^{2} D_{\mathrm{wk}} \int_{\Gamma^{\prime}} \mathbf{V} \cdot \mathbf{n}^{\prime} d \Gamma \\
& =\left\langle S_{w}\right\rangle \partial_{p} \bar{\omega} .
\end{aligned}
$$

The last step stems from the fact that the integral $\int_{\Gamma^{\prime}} \mathbf{V} \cdot \mathbf{n}^{\prime} d \Gamma$ is equal to the large-scale convergence over the grid cell $S_{t} \partial_{p} \bar{\omega}$ and that $S_{t} \pi r^{2} D_{\mathrm{wk}}$ is equal to the expectation value $\left\langle S_{w}\right\rangle$.

Then in the approximation where the integral $\int_{\Gamma^{\prime}} \mathbf{V} \cdot \mathbf{n}^{\prime} d \Gamma$ and $S_{w}$ are equal to their expectation values, Eq. (8) is obtained.

\section{APPENDIX B}

\section{Expectation Value $\widetilde{L_{\Gamma}}$ of the Wake Contour Length $\widehat{L_{\Gamma}}$, Conditioned on the Presence of Some Contour within the Grid Box}

Let $P_{0}$ be the probability that no wake contour intersects the grid box. Then, the density of probability of $\widehat{L_{\Gamma}}$ conditioned on the presence of some wake contour in the grid box reads $P\left(\widehat{L_{\Gamma}} / \widehat{L_{\Gamma}} \neq 0\right)=P\left(\widehat{L_{\Gamma}}\right) /\left(1-P_{0}\right)$. Integrating this equality multiplied by $\widehat{L_{\Gamma}}$ yields a relation between the conditional expectation value $\widetilde{L_{\Gamma}}$ of $\widehat{L_{\Gamma}}$ and the unconditional expectation $L_{\Gamma}$ :

$$
\widetilde{L_{\Gamma}}=\frac{L_{\Gamma}}{1-P_{0}} .
$$

Let $\mathcal{A}$ be the domain spanned by the centers of wakes intersecting the grid box. Here $P_{0}$ is simply the probability that no wake center is in $\mathcal{A}$. For a uniform distribution of the wake centers with density $D_{\mathrm{wk}}$, the number $n$ of wake centers in $\mathcal{A}$ follows a Poisson law with mean $D_{\mathrm{wk}} S_{A}$, which yields

$$
P_{0}=\exp \left(-D_{\mathrm{wk}} S_{A}\right)
$$

\section{REFERENCES}

Barnes, G. M., and K. Sieckman, 1984: The environment of fastand slow-moving tropical mesoscale convective cloud lines. Mon. Wea. Rev., 112, 1782-1794.

Bryan, G., D. Ahijevych, C. Davis, S. Trier, and M. Weisman, 2005: Observations of cold pool properties in mesoscale convective systems during BAMEX. Preprints, 11th Conf. on Mesoscale Processes, Albuquerque, NM, Amer. Meteor. Soc., JP5J.12. [Available online at http://ams.confex.com/ams/pdfpapers/ 96718.pdf.]

Charba, J., 1974: Application of gravity current model to analysis of squall-line gust front. Mon. Wea. Rev., 102, 140-156.

Cruette, D., A. Marillier, J. L. Dufresne, J. Y. Grandpeix, P. Nacas, and H. Bellec, 2000: Fast temperature and true airspeed measurements with the Airborne Ultrasonic AnemometerThermometer (AUSAT). J. Atmos. Oceanic. Technol., 17, 1020-1039.

Diongue, A., J.-L. Lafore, J.-L. Redelsperger, and R. Roca, 2002: Numerical study of a Sahelian synoptic weather system: Initiation and mature stages and its interactions with the large-scale dynamics. Quart. J. Roy. Meteor. Soc., 128, 18991927.

Emanuel, K. A., 1991: A scheme for representing cumulus convection in large-scale models. J. Atmos. Sci., 48, 2313-2335.

_-1993: A cumulus representation based on the episodic mixing model: The importance of mixing and microphysics in predicting humidity. The Representation of Cumulus Convection in Numerical Models, Meteor. Monogr., No. 46, Amer. Meteor. Soc., 185-192.

, 1994: Atmospheric Convection. Oxford University Press, 580 pp.

_ 1997: Overview of atmospheric convection. The Physics and Parameterization of Moist Atmospheric Convection, R. K. Smith, Ed., Kluwer, 1-28.

__ and M. Zivkovic-Rothman, 1999: Development and evaluation of a convection scheme for use in climate models. $J$. Atmos. Sci., 56, 1766-1782.

Grandpeix, J.-Y., J.-P. Lafore, and F. Cheruy, 2010: A density current parameterization coupled with Emanuel's convection scheme. Part II: 1D simulations. J. Atmos. Sci., 67, 897-921.

Haman, K. E., 1976: On the airflow and motion of quasi-steady convective storms. Mon. Wea. Rev., 104, 49-56.

Hourdin, F., and Coauthors, 2006: The LMDZ4 general circulation model: Climate performance and sensitivity to parameterized physics with emphasis on tropical convection. Climate Dyn., 27, 787-813.

Jabouille, P., J. L. Redelsperger, and J. P. Lafore, 1996: Modification of surface fluxes by atmospheric convection in the TOGA COARE region. Mon. Wea. Rev., 124, 816-837.

Jorgensen, D. P., and M. A. LeMone, 1989: Vertical velocity characteristics of oceanic convection. J. Atmos. Sci., 46, 621-640.

Khairoutdinov, M., and D. Randall, 2006: High-resolution simulation of shallow-to-deep convection transition over land. J. Atmos. Sci., 63, 3421-3436. 
Lafore, J. P., and M. W. Moncrieff, 1989: A numerical investigation of the organization and interaction of the convective and stratiform regions of tropical squall lines. J. Atmos. Sci., 46, 521-544.

— J. L. Redelsperger, and G. Jaubert, 1988: Comparison between a three-dimensional simulation and Doppler radar data of a tropical squall line: Transport of mass, momentum, heat, and moisture. J. Atmos. Sci., 45, 3483-3500.

Laing, A. G., and J. M. Fritsch, 1997: The global population of mesoscale convective complexes. Quart. J. Roy. Meteor. Soc., 123, 389-405.

LeMone, M. A., and E. J. Zipser, 1980: Cumulonimbus vertical velocity events in GATE. Part I: Diameter, intensity and mass flux. J. Atmos. Sci., 37, 2444-2457.

Moncrieff, M. W., 1981: A theory of organized steady convection and its transport properties. Quart. J. Roy. Meteor. Soc., 107, 29-50.

_, 1992 : Organized convective systems: Archetypal dynamical models, mass and momentum flux theory, and parameterization. Quart. J. Roy. Meteor. Soc., 118, 819-850.

- , and D. W. K. So, 1989: A hydrodynamical theory of conservative bounded density currents. J. Fluid Mech., 198, 177-197.

Montmerle, T., J. P. Lafore, and J. L. Redelsperger, 2000: A tropical squall line observed during TOGA COARE: Extended comparisons between simulations and Doppler radar data and the role of midlevel wind shear. Mon. Wea. Rev., 128, 3709-3730.

Piriou, J. M., J. L. Redelsperger, J. F. Geleyn, J. L. Lafore, and F. Guichard, 2007: An approach for convective parameterization with memory: Separating microphysics and transport in grid-scale equations. J. Atmos. Sci., 64, 4127-4139.

Qian, L., G. S. Young, and W. M. Frank, 1998: A convective wake parameterization scheme for use in general circulation models. Mon. Wea. Rev., 126, 456-469.

Redelsperger, J. L., F. Guichard, and S. Mondon, 2000: A parameterization of mesoscale enhancement of surface fluxes for large-scale models. J. Climate, 13, 402-421.
A. Diongue, A. Diedhiou, J.-P. Ceron, M. Diop, J.-F. Gueremy, and J.-P. Lafore, 2002: Multi-scale description of a Sahelian synoptic weather system representative of the West African monsoon. Quart. J. Roy. Meteor. Soc., 128, 1229-1258.

Rio, C., F. Hourdin, J.-Y. Grandpeix, and J.-P. Lafore, 2009: Shifting the diurnal cycle of parameterized deep convection over land. Geophys. Res. Lett., 36, L07809, doi:10.1029/ 2008 GL036779.

Rotunno, R., J. B. Klemp, and M. L. Weisman, 1988: A theory for strong, long-lived squall lines. J. Atmos. Sci., 45, 463-485.

Rozbicki, J. J., G. Young, and L. Qian, 1999: Test of a convective wake parameterization in the single-column version of CCM3. Mon. Wea. Rev., 127, 1347-1361.

Smull, B. F., and R. A. Houze, 1987a: Dual-Doppler radar analysis of a midlatitude squall line with a trailing region of stratiform rain. J. Atmos. Sci., 44, 2128-2149.

$\longrightarrow$, and — 1987b: Rear inflow in squall lines with trailing stratiform precipitation. Mon. Wea. Rev., 115, 2869-2889.

Thorpe, A. J., M. J. Miller, and M. W. Moncrieff, 1982: Twodimensional convection in non-constant shear: A model of midlatitude squall lines. Quart. J. Roy. Meteor. Soc., 108, 739-762.

Tompkins, A., 2001: Organization of tropical convection in low vertical wind shears: The role of cold pools. J. Atmos. Sci., 58, $1650-1672$.

von Kármán, T., 1940: The engineer grapples with non-linear problems. Bull. Amer. Math. Soc., 46, 615-683.

Weisman, M. L., and J. B. Klemp, 1982: The dependence of numerically simulated convective storms on vertical wind shear and buoyancy. Mon. Wea. Rev., 110, 504-520.

Yang, G.-Y., and J. Slingo, 2001: The diurnal cycle in the tropics. Mon. Wea. Rev., 129, 784-801.

Zipser, E. J., 1977: Mesoscale and convective-scale downdrafts as distinct components of squall-line circulation. Mon. Wea. Rev., $\mathbf{1 0 5}, 1568-1589$. 